\title{
The Electron Transport Chain of the Microbicidal Oxidase of Phagocytic Cells and Its Involvement in the Molecular Pathology of Chronic Granulomatous Disease
}

\author{
Anthony W. Segal \\ Department of Medicine, Faculty of Clinical Science, University College London, London, United Kingdom WC1E 6JJ
}

\section{Introduction}

The "professional" phagocytic cells, neutrophils, monocytes, macrophages, and eosinophils, demonstrate markedly enhanced oxygen consumption when engulfing their prey. This "extra respiration of phagocytosis" was first observed in 1933 (1) but the unusual nature of the process was only revealed in 1959 when it was discovered that it was not inhibited by classical mitochondrial poisons such as cyanide and azide (2), indicating that it was not simply a reflection of the enhanced energy requirements of phagocytosis. This "respiratory burst" was soon discovered to be a requirement for the efficient killing of bacteria by neutrophils (3), the veracity of this observation being rapidly reinforced by the identification of a new syndrome, "fatal granulomatosis of childhood" (now referred to as chronic granulomatous disease [CGD]), ${ }^{1}$ in which a severe predisposition to pyogenic infection was associated with complete absence of this oxidase activity from the patients phagocytes (4).

An extensive series of investigations have been undertaken in an attempt to define the nature of this oxidase and the molecular basis of CGD. These studies have taken three main directions: the isolation of the intact functional oxidase, the identification and purification of its components, and the reassembly of these components to reconstitute a functional system. In each case, cells from patients with CGD have played an important role in the experimental approach. This perspective article will outline the current state of knowledge that has resulted from these studies and derive a model to account for their findings and to explain the molecular pathology of CGD.

Early attempts to identify the oxidase system in cells, organelles, and extracts

The earliest experiments attempted to purify the oxidase from whole-cell homogenates and crude subcellular fractions. These were incubated with a variety of potential substrates in an attempt to demonstrate oxidase or diaphorase activity (5). The dramatic increase in the activity of the hexose monophosphate shunt in association with the respiratory burst (6) indicated one of its products, NADPH (7), as the most physiological of these substrates. A variety of activities were detected, but the

Received for publication 29 November 1988 and in revised form 13 January 1989.

1. Abbreviations used in this paper: CGD, chronic granulomatous disease; FAD, flavin adenine dinucleotide; NCF, neutrophil cytosolic factor; $66 \mathrm{~K}, 66,000-\mathrm{mol}$ wt flavoprotein.

J. Clin. Invest.

(c) The American Society for Clinical Investigation, Inc.

0021-9738/89/06/1785/09 \$2.00

Volume 83, June 1989, 1785-1793 interpretation of these experiments was complicated by the lack of specificity of the oxidation of many of these substrates and the autocatalytic nature of many of the resulting reactions. A number of "enzymes" were discovered and described as defective in CGD (5).

The next advance in this approach came with the solubilization of an active oxidase from activated cells (8). Despite this achievement, complete purification of the source of the detectible activity was prevented by the instability of the system, particularly its exquisite sensitivity to salts, which prevented separations on most chromatographic media (9). Purifications of the "oxidase" by these methods were probably separating a proximal component of the system, as will be elaborated upon below.

\section{Identification of cytochrome b-245 and recognition of the oxidase as an electron transport chain}

In 1978 a b-type cytochrome was identified in human neutrophils (10), having been previously seen in animal cells (11). This discovery seemed significant inasmuch as this was the sort of molecule that might be expected to accomplish the one electron reduction of oxygen to form superoxide, and pointed the way to the identification of the "NADPH oxidase" as an electron transport chain rather than a single enzyme.

In humans this cytochrome was found in the professional phagocytic cells, neutrophils, monocytes, macrophages, and eosinophils, but not in a variety of other cell types (12). Its identity in these different cells was established by the determination of its midpoint potential $\left(\mathrm{Em}_{7.0}\right.$, see below). It is present in myeloid HL-60 (13) and U937 (14) cell lines. In the former the induction of differentiation with dimethyl sulphoxide was associated with the development of oxidase activity and a parallel increase in the concentration of this cytochrome (13).

In these cells it is found in the plasma membrane and becomes incorporated into the wall of the phagocytic vacuole as this is formed by an invagination of this membrane (15). In neutrophils an additional pool of the cytochrome is detected in the membrane of the specific granules which also transfers to the membrane of the phagocytic vacuole (16).

BIOCHEMICAL PROPERTIES

Spectroscopy in alkaline pyridine reveals the spectrum of a protoheme pyridine hemochrome characteristic of a b-type cytochrome (17), which is present in neutrophils and monocytes at a concentration of $\sim 100 \mathrm{pmol} / \mathrm{mg}$ protein (18). The function of a redox molecule is largely dependent upon its midpoint potential (19), at which it is balanced between oxidation and reduction, which governs its bioenergetic stature in the heirarchy of components of an electron transport chain. At $245 \mathrm{mV}(20)$ this cytochrome has the lowest midpoint potential of any mammalian cytochrome $b$, which provides it with the capability of directly reducing oxygen to superoxide (21). This measurement gives a considerable degree of discrimina- 
tion between otherwise apparently similar molecules and it is for this reason that we refer to it as cytochrome $b$-245. It is also called "cytochrome $b 558$," the 558 referring to the wavelength of its $\alpha$ band of light absorption.

Another unusual property of this cytochrome, not seen in other mammalian cytochromes $b$ but shared with bacterial cytochromes $o(22)$, is that it binds $\mathrm{CO}(20,23)$ (recombination time after flash dissociation of the complex of $\sim 6 \mathrm{~ms}$ ). This property indicates that it probably binds oxygen (the oxidation of the reduced cytochrome is very rapid with a half-time of 4.7 ms [23]) and represents the terminal component of the electron transport chain.

NADPH reduces the cytochrome in preparations of the solubilized oxidase, but only at a very slow rate. This slow rate of reduction had been thought by some (24) to preclude its participation as a component of the oxidase. The kinetics of reduction of a molecule in an electron transport chain under anaerobic conditions might bear no relationship to its normal physiological function in the presence of an electron acceptor which can help to pull electrons through the system. When the steady-state kinetics of reduction of the cytochrome were determined in the presence of oxygen, they correlated almost exactly with the observed rate of $\mathrm{O}_{2}^{-}$generation (25).

PURIFICATION AND IDENTIFICATION OF CYTOCHROME $b$-245 The purification of this molecule and identification of its apoprotein polypeptide proved difficult. The four initial purification schemes described isolated polypeptides of different molecular weights, varying between 11,000 and 127,000 (26-29). The main causes of these difficulties in the identification of the molecule were proteolysis, the neutrophil being richly endowed with a wide range of potent proteolytic enzymes, and anomalous behavior of the apoprotein(s) on sodium dodecyl sulfate-polyacrylamide gel electrophoresis (SDS-PAGE) (30).

Two reliable methods now exist for the purification of this cytochrome, both of which resulted in the purification of not one but two proteins, with molecular weights of 76,000 92,000 and 23,000 as determined by mobility on SDS-PAGE.

\section{SUBUNIT STRUCTURE}

The association of the 23,000-mol wt $\alpha$ and 76,000-92,000mol wt $\beta$ subunits has been established by their copurification with the heme of the cytochrome; their association on gel filtration chromatography, sucrose density, and pH gradients; cross-linking studies; and the absence of both proteins from cells of patients with X-linked CGD (31-33). A number of these heterodimers might be associated to form a macromolecular complex.

The larger $\beta$ subunit migrates on SDS-PAGE as a broad band, an electrophoretic property characteristic of glycoproteins, with an apparent molecular mass of $\sim 60-90 \mathrm{kD}$. It was therefore not surprising when this molecule was shown to be heavily glycosylated (30) with about $21 \%$ carbohydrate, predominantly of the $\mathrm{N}$-linked high-lactosamine complex-type oligosaccharide moieties.

The two subunits are very tightly associated and any maneuvers designed to separate them also displace the heme. Thus, we still do not know which subunit binds the heme, or whether it is located between these subunits.

The genes for both these molecules have been cloned and sequenced $(34,35)$, that for the $\beta$ subunit through the innovative application of reverse genetics as described below. Unfortunately, knowledge of their amino acid sequence throws little light on their function, with no obvious homology with any other described protein. The minimal homology claimed between the $\alpha$ subunit and mitochondrial cytochrome $c$ oxidase (35) is of doubtful relevance. Transcription of the larger $\beta$ subunit is largely confined to the myeloid cell lineage and is increased in parallel with expression of the cytochrome after the induction of oxidase activity in cells of patients with variant CGD by interferon- $\gamma$ (IFN- $\gamma$; see below).

The apparent transcription of the $\alpha$ subunit in a number of cell types other than phagocytic cells, which do not demonstrate oxidase activity or spectral evidence of cytochrome $b-245$ (35), is particularly interesting. Hybridization between the clone and a similar but not identical RNA is one explanation of these findings, although S1 nuclease was employed in order to protect against this possibility.

In addition, unlike oxidase activity and spectral activity of the cytochrome $b$, expression of the $\alpha$-subunit is not enhanced by INF- $\gamma$ (36). Thus, even though most cytochromes $b$ are roughly of the size of the 23,000 -mol wt $\alpha$-subunit, and on the grounds of size alone it would appear the most likely candidate as the sole heme binding molecule, the evidence is against this. The continuous transcription of an "orphan" molecule in the absence of a demonstrable product of translation or attributed function seems unacceptably wasteful. It could be a constitutive protein playing a controlling or structural role in relation to the $\beta$ subunit of the cytochrome, and have a similar function in different processes in the other tissues in which it is transcribed. The reported failure to detect the product of translation in these tissues (36) could result from low steadystate concentrations as a result of rapid turnover of the molecules, or to practical experimental problems.

\section{Involvement of cytochrome b-245 in CGD}

CGD is a syndrome in which absence of this NADPH oxidase system is associated with an unusual predisposition to infection (4). A number of different patterns of inheritance are found, indicating different genetic mechanisms and molecular lesions (37-39). After having discovered this cytochrome $b$, it was of considerable interest to determine whether its absence was responsible for the defective function of the electron transport chain in cells from these patients.

With few exceptions the cytochrome has been found by spectral analysis to be missing from cells of subjects with the more common inheritance through a lesion on the $\mathrm{X}$ chromosome and normal in those with an autosomal recessive pattern $(18,37,38)$. In addition, both subunits of the cytochrome were shown to be absent from the patients' cells in X-linked CGD (31), confirming the association of these proteins as subunits of the cytochrome, but providing no indication as to the nature of the genetic lesion.

\section{Cloning of the gene for the $\beta$-subunit of cytochrome b-245}

The genetic lesion in most patients with X-linked CGD was identified through the elegant application of "reverse genetics" (34). DNA from a very unusual patient with a deletion of a small segment of the short arm of the $\mathrm{X}$ chromosome was used in a subtractive hybridization to enrich in normal DNA from the region of the deletion. This DNA was cloned (40) and the library probed with cDNA prepared from (34) an induced HL-60 cell line subtractively hybridized with RNA from another CGD patient with a small deletion on the short arm of the $\mathrm{X}$ chromosome. Clones were identified that hybridized 
with RNA with a 5-kb message, showed the appropriate tissue specificity and were induced in HL-60 cells in association with induction of the oxidase. Confirmation of the connection between this gene (labeled the "X-CGD gene") and the oxidase was supplied by the demonstration that monocytes from three of four X-linked CGD patients failed to transcribe complementary RNA. Although cells from a fourth such patient did transcribe the gene, it showed an abnormal pattern of cleavage by restriction enzymes, the patient's mother being heterozygous for this abnormality.

In having identified the abnormal gene by these methods, the protein for which it coded was predicted but not identified. In particular, it did not show sequence homology with cytochromes $b$.

Starting with the pure cytochrome, we were able to dissociate the two subunits by denaturation and then separate them by gel filtration. The sequence of 42 amino acids from the amino terminus of the $\beta$ chain of cytochrome $b-245$ was then determined. The corresponding DNA sequence was identified in the X-CGD gene, in a region originally designated as noncoding as a result of a nucleotide sequencing error (41), adding 101 amino acids to the translation product with a predicted molecular weight of 65,000 . This observation, together with binding studies with an antibody made to the fusion protein product of their gene (42), unequivocally identified the primary lesion in these patients in the gene coding for the $\beta$ chain of cytochrome $b-245$. Of interest is the observation that the small $\alpha$ subunit is undetectable in the cells of patients with $X$-linked CGD (35), a requirement of the $\beta$ subunit in the transcription, translation, or stability of this molecule.

A few cases of CGD have been described where a convincing X-linked pattern of inheritance is associated with detectable cytochrome $b$. In some of these patients there are normal amounts of the cytochrome associated with some, albeit very slow electron transport down the chain (43). In others, the so-called "variant" cases, a reduced level of oxidase activity is seen with variable reductions in the cellular concentration of this molecule (44). It is inevitable that a variety of mutations in the CGD gene, resulting in different mechanisms of malfunction of the cytochrome, will be defined in the future.

\section{Flavoproteins}

It is highly likely that a flavoprotein is a, if not the, component linking the substrate, NADPH, and cytochrome $b-245$. The involvement of a flavoprotein in this oxidase was postulated by Cagan and Karnovsky in 1958 (45), and there is a lot of evidence to suggest that this is correct and that the cofactor is flavin adenine dinucleotide (FAD) $(5,7,8)$. The activity of a solubilized preparation of the oxidase is inhibited by flavin analogues like 5-carba-deaza-FAD (46) and the possible flavoprotein inhibitor diphenylene iodonium (47). A fluorescent flavoprotein has been described in neutrophil membranes (48). FAD in membranes and membrane extracts is reduced by NADPH under anaerobic conditions $(49,50)$, and a flavin semiquinone electron spin resonance spectrum has been observed in membranes from activated neutrophils in the presence of NADPH (51).

A number of groups have approached the identification of the oxidase by solubilizing and purifying oxidase activity, and have isolated a protein with a molecular weight of $\sim 65,000$ 67,000 , which in most cases appears to be a flavoprotein $(9,52$, 53). Unfortunately, the oxidase activity is very unstable so that the recovered activity of $\sim 5 \%(9,52)$ can leave the identity of the final product in some doubt.

There is some evidence to suggest that this $65,000-67,000$ mol wt flavoprotein (henceforth referred to as $66 \mathrm{~K}$ ) binds NADPH. 2',3'-Dialdehyde NADPH and sodium cyanoboro $\left[{ }^{3} \mathrm{H}\right]$ hydride was used to label neutrophil membranes and a strong band of labeling was observed with a molecular weight of 66,000 (53). Similar experiments with a photoaffinity NADPH-labeling procedure appeared to identify these as the same protein (54).

An important feature of this molecule that has caused considerable confusion and resulted in reluctance to accept the participation of the cytochrome $b$ as a component of the oxidase $(24,52,53)$ is its ability to transport electrons directly from NADPH to other acceptors, including cytochrome $c$, which is used in the assay of superoxide generating activity. It is likely that in methods designed to purify the oxidase, the electron transport chain disintegrates, leaving the proximal flavoprotein component, which appears to be the only one with the capability of binding the substrate NADPH, as the only identifiable molecule. Thus, detectable electron transferring activity probably moves from the complete system to the flavoprotein during purification. The flavoprotein alone is likely to be much less efficient in the generation of superoxide than the integrated electron transport chain, and thus the purification of this molecule rather than the complete system could be one of the factors responsible for the very low recoveries of activities in these purifications $(9,52)$. The transition from the generation of superoxide to the direct transfer of electrons to cytochrome $c$ (52) might be due to direct access of this electron acceptor to the active site of the dehydrogenase after the removal of membrane lipids by detergents. Partial regeneration of superoxide production by the addition of lipid to the system (52) could result from stearic hindrance to this direct interaction inducing the requirement for the one electron reduction of product of oxygen, superoxide, to act as a redox intermediate.

Sakane and colleagues have described another NADPH flavoprotein in these cells which appears to be cytochrome $\mathrm{P}-450$-reductase (55) and could be unrelated to the microbicidal oxidase system.

\section{Flavoproteins in CGD}

The 66,000-mol wt, membrane-related NADPH-binding protein has been shown to be present in cells of patients with $\mathrm{X}$-linked and with autosomal recessive CGD (56). This was an interesting finding because flavoprotein concentrations of about half-normal have been detected in membranes from neutrophils in a large proportion of the cases of X-linked CGD $(37,38,48,57)$, suggesting the participation of a flavoprotein other than the $66 \mathrm{~K}$ in this system. The absence of this protein in association with that of the cytochrome $b$ might suggest the same sort of structural relationship between these molecules as appears to exist between the 47,000-mol wt phosphoprotein (described below) and the cytochrome $b$ (58).

\section{7,000-mol wt phosphoprotein and its abnormality in CGD}

Although cells from these patients contain normal amounts of an apparently normal cytochrome $b(18)$, they are unable to pass electrons along the chain onto this cytochrome (59). This indicates an abnormality of the activation process or the ab- 
sence or malfunction of a proximal electron transferring molecule, or molecules.

Activation mechanisms in neutrophils (7) are currently the focus of considerable interest. One of the major common pathways appearing to be through the phosphorylation of the target protein by protein kinase $C$, which is directly activated by phorbol myristate acetate (PMA), a potent stimulus of the oxidase system (60).

It thus seemed pertinent to determine the pattern of protein phosphorylation in cells from these patients after activation with PMA, the hypothesis being that a general abnormality of activation would be identifiable by the failure to phosphorylate a number of bands and a defect of a specific target would be revealed by a single missing or aberrant band. In fact, the second possibility proved correct with the consistent absence of a 47,000-mol wt phosphoprotein from the cells of these patients (61-63).

The normal protein, but not that from the cells of these patients, could be phosphorylated by protein kinase $C$ in vitro after extraction from SDS-PAGE gels (64). This could indicate that the amino acid target of phosphorylation, usually serine or threonine, has been replaced as a result of a base substitution in the gene, but the evidence suggests the protein itself to be missing (65).

This 47,000-mol wt phosphoprotein is located predominantly in the cytoplasm and translocates into the membranes upon activation of the oxidase $(58,64,66)$. Of particular interest was the observation that this translocation fails to occur in cells from patients with X-linked CGD from which the cytochrome $b$ was missing (58). This indicates that the 47,000mol wt phosphoprotein does not move randomly into the membranes and then asssociate with the electron transport chain, but that its membrane binding is dependent upon attachment to the cytochrome, or an associated molecule.

\section{Other components of the electron transport chain}

The other main types of electron transporting molecules are quinones and nonheme iron proteins. Some investigators have identified ubiquinone in extracts of neutrophils and claim it as a component of the electron transport chain $(67,68)$. Apart from its role in this oxidase being thermodynamically improbable because of its relatively high midpoint potential, the ubiquinone detected appears to have been derived from the mitochondria of contaminating platelets and mononuclear cells (69). Nonheme iron proteins have not been detected in neutrophil membranes (20). One other protein that might be involved has a molecular weight of $\sim 32,000$. Under certain circumstances it appears to copurify with cytochrome $b-245$ (8). It is also 1 of at least 10 proteins visible in a "purified respiratory burst oxidase" isolated by Babior's group in which those with molecular masses of 66,45 , and $32 \mathrm{kD}$ have been singled out for consideration as likely subunits (70). Curiously, the same purification was initially described as yielding only one major band with a molecular mass of $65 \mathrm{kD}(9)$.

Reconstitution of a functional oxidase by the recombination of neutrophil subcellular compartments

An advance in our understanding of the oxidase was achieved by the discovery that the system could be induced to function in a "cell-free" system of disrupted constituents of unactivated cells by the addition of a detergent, SDS (71) and. later by arachidonic acid $(72,73)$. Subsequently, separated membranes and membrane extracts, and cytosol could be recombined to restore function, identifying functional components of this system in both these cellular compartments $(35,73-77)$.

A functional system could not be reassembled when CGD cells were used. By mixing of normal membranes and cytosol with those from patients, it was possible to localize the site of their lesions. Predictably, in X-linked CGD the absence of the b-type cytochrome was associated with a defect in the membrane fraction, whereas, patients with autosomal recessive CGD in whom the $47-\mathrm{kD}$ band of protein did not become phosphorylated, had abnormal cytosol $(63,78,79)$.

Kinetic data suggested the requirement for more than one cytosolic factor (76), and this was confirmed when the cytosolic requirements of this reconstituted system were further defined after separation of the proteins on an anion exchange column (63). It was shown that three components could be defined, called neutrophil cytosol factor 1-3 (NCF), in their order of elution from the column, which together could replace the cytosol in the reconstituted system. NCF-1 could correct the defect in the cytosol of all but one of the autosomal recessive patients. NCF-2 corrected the cytosolic deficiency in the single patient whose cell extracts did not respond to NCF-1 and who was shown to have a different anomaly on the basis of complementation studies. Unlike all the others, the 47,000mol wt phosphoprotein in his cells was normally phosphorylated upon activation. Independently, another group of investigators had raised an antiserum to cytosolic proteins eluted from a GTP affinity column which reconstituted activity, which resulted in antibodies predominantly to proteins with molecular weights of 47,000 and 65,000 (80). Rather fortuitously, these 47,000- and 65,000-mol wt proteins were located in the NCF-1 and NCF-2 fractions, respectively, and Western blotting studies indicated that the autosomal recessive patients lacking NCF-1 and NCF-2 activity were also missing the corresponding protein from their cytosol $(63,80)$.

These studies indicate that most autosomal recessive patients are lacking a cytosolic factor, NCF-1, almost certainly the 47,000 phosphoprotein. NCF-2 appears to be a protein with a molecular weight of 65,000 , very close to that of the putative NADPH-binding flavoprotein. This might suggest that NCF-2 and the 66,000 -mol wt flavoprotein are one and the same. However, this seems unlikely in that the former is cytosolic, moving into the membranes upon activation, whereas the latter has been clearly identified within the membrane fraction of unstimulated neutrophils $(53,56)$ and their pIs appear to differ (H. Malech, personal communication). It is important to note that although the $66,000-\mathrm{mol}$ wt membrane protein described above appears to bind NADPH, there is no direct evidence linking it to the oxidase system, whereas, the absence of the $65,000-\mathrm{mol}$ wt cytosolic protein in a patient with autosomal recessive CGD together with its presence in fractions of cytosol that reconstitute oxidase activity in the "cell-free system" implicate this molecule as an important participant.

One other cytosolic component, designated NCF-3, which elutes late from the anion exchanger that is required together with NCF-1 and -2 for the replacement of cytosolic activity in the reconstituted system remains to be identified. A note of caution is required in the interpretation of results of these reconstituted systems. Redox components with the appropriate midpoint potential can substitute for the natural molecule. Thus, for example, the demonstration that a purified dehydrogenase can induce the reduction of cytochrome $b-245$ 
does not necessarily mean that this must be the proximal electron donor. In this case it could well by cytochrome P-450 reductase (81).

\section{Influence of IFN- $\gamma$ on the oxidase and its components}

IFN- $\gamma$ has been shown to enhance the oxidase response of normal phagocytes (82). A major advance was made in the use of cytokines to the treatment of human disease when it was shown that this agent had a corrective effect on phagocyte function in CGD (83).

The studies on the influence of IFN- $\gamma$ on CGD phagocytes have taken three main directions: the activity of the oxidase in relationship to the cytochrome $b$; transcription and translation of the genes for the subunits of the cytochrome; and the effect of treatment on bacterial killing. Sechler and colleagues (79) observed the effect of IFN- $\gamma$ on oxidase activity of cultured monocytes. All but 1 of the 13 autosomal recessive subjects whose cells contained cytochrome $b$ showed increased activity, from undetectable levels to $\sim 14 \%$ of normal. The autosomal recessive patient without the cytochrome did not demonstrate activity before or after treatment. X-linked cytochrome $b$-deficient subjects clearly fell into two groups: 9 of the 13 showed no response; restitution of activity was partial in three and complete in the remaining one. An incomplete response was also seen in the unusual X-linked patient whose cells contained the cytochrome. "Variant" patients with an incomplete defect also demonstrated improved activity. The response was maximal at a concentration of IFN- $\gamma$ of about $1,000 \mathrm{U} / \mathrm{ml}$ and evolved over $\sim 48 \mathrm{~h}$. In vivo responses in neutrophils and monocytes were of the same order of magnitude as seen in vitro and also took $\sim 3 \mathrm{~d}$ to reach maximum.

These changes in oxidase activity were reflected by changes in the expression of the genes for, and the tissue levels of, cytochrome $b-245$ (36). Incubation with IFN- $\gamma$ markedly increased the mRNA transcript for the large $\beta$ subunit of the cytochrome in neutrophils, monocytes, and cell lines of the myeloid lineage to about three to five times the levels in untreated cells. These changes were maximal after $\sim 24-48 \mathrm{~h}$. Transcript levels for the light $\alpha$ subunit were unchanged. $\mathrm{Nu}$ clear runoff studies demonstrated accelerated transcription rather than delayed removal as the basis of the accumulated message for the $\beta$ subunit.

Tumor necrosis factor has been shown to have similar effects to IFN- $\gamma$, and to act synergistically with it, on the gene for the $\beta$ subunit and on the expression of oxidase activity (84). The effects of these mediators on oxidase activity and mRNA levels for the $\beta$ subunit of the cytochrome are mirrored by cellular levels of cytochrome $b$, as measured spectrophotometrically or immunologically.

The changes in oxidase activity in neutrophils and monocytes that resulted from the administration of IFN- $\gamma$ to patients with CGD were associated with marked improvements in bacterial killing, which virtually returned to normal at levels of oxidase activity at $\sim 10 \%$ of normal (79). Interestingly, killing by neutrophils of some patients was enhanced in the absence of detectable induction of superoxide generation, suggesting some other influence on the biology of these cells, whose microbicidal capability is not solely related to the oxidase system, as will be discussed below.

Given the dramatic response in a number of these patients to exogenous IFN- $\gamma$, it is interesting to consider why the same changes are not seen as a result of the endogenous secretion of this compound in the setting of chronic inflammation that most of them sustain. Other cytokines are also secreted under these conditions and might compete with IFN- $\gamma$ and downregulate this system. In this context IFN- $\alpha$, which suppresses the respiratory burst, has been shown to reduce transcription of the gene for the $\beta$ subunit of the cytochrome (36).

$A$ unifying model of the oxidase electron transport chain and its relationship with the different subtypes of CGD

On the basis of the information outlined above it is possible to construct a model of the oxidase system and to postulate the molecular basis for the different subgroups of CGD (Fig. 1). Cytochrome $b-245$ is almost certain to represent the terminal component of the chain. It appears to bind oxygen, the final electron acceptor, and has a rapid oxidation rate in keeping with a terminal oxidase and a sufficiently low midpoint potential to permit the direct reduction of oxygen to superoxide. Glycosylation of the $\beta$ subunit suggests a location on the external surface of the plasma membrane for this molecule, the surface from which the superoxide is released. The location of the smaller $\alpha$ subunit is unknown. Both subunits are phosphorylated upon stimulation of the oxidase, suggesting a requirement for changes in conformation to achieve the active state.

The 47,000-mol wt phosphoprotein is cytosolic and is phosphorylated upon activation of the cell. It then moves into the membranes where it attaches to a binding site on the cytochrome $b$. It might itself be an electron-transporting mole-

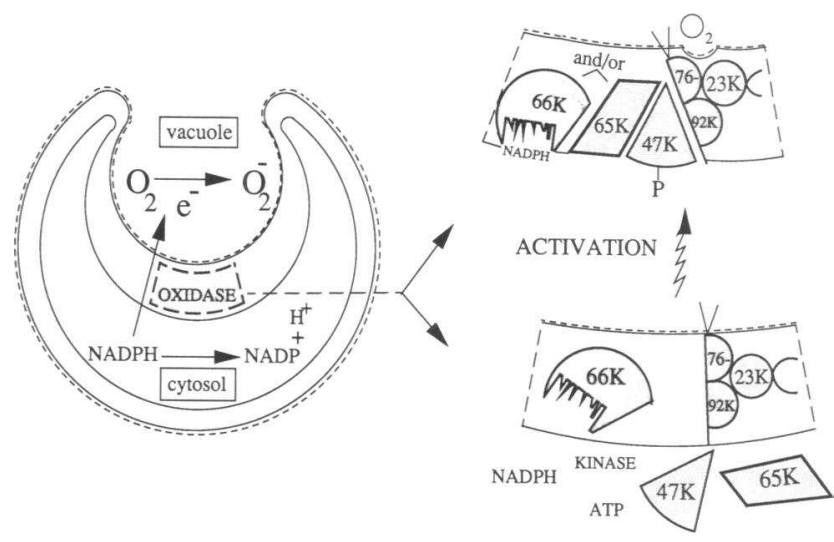

Figure 1. A schematic representation of a model of the NADPH oxidase of phagocytic cells. The oxidase consists of an electron transport chain that is interposed between the substrate, NADPH, in the cytosol, and the lumen of the phagocytic vacuole. Electrons are pumped across this membrane to reduce oxygen to superoxide, thereby elevating the $\mathrm{pH}$ within the vacuole. The clearly defined components of this chain include cytochrome $b-245$, with a large $\beta$ and a 28,000mol wt $\alpha$ subunit, and a 47,000-mol wt cytosolic protein that, upon activation of the system, becomes phosphorylated by a kinase using ATP as substrate, and moves into close association with the cytochrome in the membrane. The proximal molecule could be a flavoprotein with a molecular weight of $65,000-67,000$ located in the membrane, representing a 66,000 -mol wt NADPH-binding protein located in the membrane from the outset, or a $65,000-\mathrm{mol}$ wt cytosolic factor that moves into the membrane upon activation, or possibly both. In most cases, $X$-linked CGD results from a defect in the gene coding for the $\beta$ subunit of the cytochrome, whereas in the majority of those with the autosomal recessive inheritance the lesion affects the 47,000-mol wt phosphoprotein. $\mathrm{K}$, molecular weight in thousands. 
cule or could play a structural or regulatory role in the integration or activation of the chain. Stimulation of neutrophils is followed by variable delay before triggering of the NADPH oxidase. This takes $\sim 20-30 \mathrm{~s}$ after the addition of the C-kinase activator PMA, but is much more rapid after exposure to the chemotactic peptide FMLP (85). The latter may trigger the burst without obvious phosphorylation of the 47,000-mol wt phosphoprotein (86). It is possible that the lag phase reflects the time taken for phosphorylation of this protein, its translocation into the membrane, and its association with the cytochrome $b$. The interaction of FMLP with its receptor might induce the integration of the components of the chain without the requirement for phosphorylation. The failure of FMLP to activate the oxidase in autosomal recessive CGD in which the 47,000-mol wt phosphoprotein is defective indicates the requirement for this component whether or not phosphorylation is observed.

There is clearly a requirement for a $65,000-\mathrm{mol}$ wt cytosolic factor that must move into the membranes upon activation of the system. Whether this is the proximal NADPHbinding flavoprotein or it intercalates into the chain distal to a 66,000 -mol wt membrane protein with these characteristics remains to be resolved.

It has been found that this system can be "primed" by the exposure of cells to concentrations of stimuli below those required for activation of the oxidase. This priming, which results in a decrease in the lag before activation, and amplifies the response, could involve phosphorylation and partial assembly of the components of the chain.

\section{Models for the different types of CGD}

The defective gene in X-linked CGD is that coding for the large $\beta$ subunit of the cytochrome $b$. The most common result of an abnormality of this gene is the failure of transcription of its message, as well as that of the smaller $\alpha$ subunit, and the complete absence of both proteins from the patients' phagocytes. The genetic lesion is likely to preclude any expression of the cytochrome in the majority of these patients in whom the oxidase system shows no response to IFN- $\gamma$ (79).

A minority of these patients in whom cytochrome $b$ was undetectable before treatment with IFN- $\gamma$ did respond to this agent by the generation of superoxide. The levels of the cytochrome were not measured in the responding cells. It is likely that these patients fall into the extreme end of the spectrum which also includes the so-called variant condition in which the cytochrome is present in lowered, but differing, amounts. These conditions are probably caused by point mutations in this gene resulting in a wide spectrum of abnormalities of the molecule, including splicing errors, an unstable protein, structural disorganization that results in impaired binding of the $a$ subunit, 47,000-mol wt phosphoprotein, and other related components of the chain. About $5-10 \%$ of the total cytochrome content of normal neutrophils is due to cytochromes like those in mitochondria and cytochrome P-450 in the endoplasmic reticulum and thus it is impossible by simple spectroscopic analysis to exclude small amounts of cytochrome $b-245$ from this background spectral activity. The response to IFN- $\gamma$ in these subjects probably reflects the markedly enhanced synthesis of the defective, but partially functional, $\beta$ subunit or limited increase in the expression of a normal molecule.

Autosomal recessive inheritance will be associated with ab- normalities of all the other proteins in the chain and the molecules responsible for their activation and integration. The most readily identifiable of these will be in those rare patients with this pattern of inheritance whose cells are missing the cytochrome $b$ in whom the lesion is most likely to affect the gene coding for the small $\alpha$ subunit.

Most of the autosomal recessive subjects have an abnormality of the 47,000-mol wt phosphoprotein, thereby blocking the transport of electrons to the cytochrome. The study by Malech's group suggests that this results from a failure of synthesis of this molecule rather than a point mutation resulting in the loss of a phosphorylation site (63). Almost without exception, cells of these patients show a small but significant response to IFN- $\gamma$. As discussed above, this agent cannot induce oxidase activity in the absence of cytochrome $b$, indicating that the other redox molecules cannot make superoxide without this cytochrome, and thus IFN- $\gamma$ must act by improving the connection between the NADPH-binding molecule and the cytochrome. It probably achieves this effect in these autosomal recessive patients by inducing the synthesis of the 47,000-mol wt protein, although it is conceivable that it could initiate the direct, albeit inefficient, transfer of electrons in the absence of this molecule.

\section{Influence of the electron transport chain on vacuolar $\mathrm{pH}$, microbial killing, and digestion}

In the light of our understanding of the structure of the oxidase as an electron transport chain, we can extend the concept of its function to a mechanism for the elevation of the $\mathrm{pH}$ of the phagocytic vacuole which has a profound effect upon killing and digestion within this compartment. It had been accepted, probably largely by analogy with the acid conditions pertaining in the secondary lysosomes of liver cells, that the phagocytic vacuoles of phagocytes are acidic. Early studies with $\mathrm{pH}$ sensitive dyes coupled to phagocytosed particles appeared to confirm this $(87,88)$, but were only detecting relatively late events. In fact, the $\mathrm{pH}$ within this compartment in normal cells undergoes a remarkable initial rise to $\sim 7.8-8.0$ before gradually drifting down to $\sim 6.0(89,90)$. This elevation is accomplished by the pumping of electrons by the electron transport chain, which has been shown to be electrogenic (91), unaccompanied by protons, into the vacuole. Within the vacuole the reduced oxygen products, superoxide and peroxide, are anionic and consume protons as they disproportionate to the protonated form.

Reduced products of oxygen are not themselves potent microbicidal agents. Cytoplasts, neutrophils from which the cytoplasmic granules and nuclei are removed by centrifugation (92), phagocytose bacteria and demonstrate a normal respiratory burst but fail to kill Staphylococci normally (93), indicating a requirement for the granule contents in the killing process.

Myeloperoxidase has been proposed as an important antimicrobial protein. The introduction of the pure enzyme into the phagocytic vacuole of cytoplasts has been shown to reconstitute microbicidal activity (93). However, myeloperoxidase is not essential for immunity to infection because large numbers of symptomless subjects have been identified whose cells are completely devoid of this enzyme (94), and it is not found in neutrophils of chickens (95).

The neutrophil granules contain proteins, particularly a group which are strongly cationic, that are potently microbici- 
dal when extracted and combined with organisms in vitro (95). The anomaly is that the same proteins are much less effective when released onto the same organism within the phagocytic vacuole of a neutrophil in the absence of oxidase activity under anaerobic conditions or in CGD. This must indicate an impairment of the action of these proteins within this environment. One way in which this might occur could be through variations in vacuolar $\mathrm{pH}$.

In CGD, and in normal cells in the absence of oxygen, the early elevation of $\mathrm{pH}$ is not observed and the $\mathrm{pH}$ falls precipitously below neutral to $\sim 1.5 \mathrm{pH}$ units lower than normal (89). This is important because it has been demonstrated that bacterial killing by the granule cationic proteins is only observed at $\mathrm{pHs}$ above 7.0 (95). This would explain the reason that CGD cells kill most, but not all ingested organisms (89). Microbes entering the vacuole early after phagocytosis will be exposed to granule contents while the $\mathrm{pH}$ is still neutral and will be killed, whereas those that are slightly more resistant or are sequestered within a clump of organisms, might survive until the $\mathrm{pH}$ has fallen to safer levels.

Not all the features of CGD can be explained in terms of their predisposition to infection. Granulomata develop in the absence of active infection and the macrophages are engorged with lipid rather than invading microbes. The phagocytic vacuoles of CGD neutrophils remain much smaller than normal $(89,96)$, and some patients develop massive unexplained hepatosplenomegaly. In fact, the original description of the histology in CGD concluded that the appearances more closely resembled a storage disease like Niemann-Pick disease than infection, but were unable to explain the mechanism by which this might occur (97). Neutrophil, monocyte, and macrophage granules contain a potent array of digestive enzymes that are largely active at neutral $\mathrm{pH}$. It was therefore predictable that the abnormal acidity of the phagocytic vacuoles in CGD would impair this function (89) and result in the accumulation of indigestable debris. In fact, CGD mirrors Niemann-Pick in being a storage disease as a result of the failure of degradation. However, in CGD this occurs not because of an absence of the hydrolytic enzyme, but rather as a consequence of the impairment of the activity of normal enzymes by an inclement milieu. This realization permits the use of maneuvers to neutralize the $\mathrm{pH}$ within the vacuoles of these patients as an additional approach to the alleviation of the manifestations of CGD. It will be of interest to see the effects of IFN- $\gamma$ and other cytokines on the indigestion normally suffered by phagocytes in these patients.

\section{Acknowledgments}

I would like to thank the Wellcome Trust, Medical Research Council and The Special Trustees of University College Hospital for support and M. C. Bohler, C. Teahan, and P. Heyworth for reading the manuscript.

\section{References}

1. Baldridge, C. W., and R. W. Gerard. 1933. The extra respiration of phagocytosis. Am. J. Physiol. 103:235-236.

2. Sbarra, A. J., and M. L. Karnovsky. 1959. The biochemical basis of phagocytosis. 1. Metabolic changes during the ingestion of particles by polymorphonuclear leukocytes. J. Biol. Chem. 234:1355-1362.

3. Selvaraj, R. J., and A. J. Sbarra. 1966. Relationship of glycolytic and oxidative metabolism to particle entry and destruction in phagocytosing cells. Nature (Lond.). 211:1272-1276.
4. Holmes, B., A. R. Page, and R. A. Good. 1967. Studies of the metabolic activity of leukocytes from patients with a genetic abnormality of phagocyte function. J. Clin. Invest. 46:1422-1432.

5. Klebanoff, S. J. 1971. Intraleukocytic microbicidal defects. Annu. Rev. Med. 22:39-62.

6. Sternholm, R., and R. C. Manak. 1970. Carbohydrate metabolism in leukocytes. X1V. Regulation of pentose cycle activity and glycogen metabolism during phagocytosis. J. Reticuloendothel. Soc. 8:550-560.

7. Rossi, F. 1986. The O2--forming NADPH oxidase of the phagocytes: nature, mechanisms of activation and function. Biochim. Biophys. Acta. 853:65-89.

8. Bellavite, P. 1988. The superoxide-forming enzyme system of phagocytes. J. Free Radicals Biol. Med. 4:225-261.

9. Markert, M., G. A. Glass, and B. M. Babior. 1985. Respiratory burst oxidase from human neutrophils: purification and some properties. Proc. Natl. Acad. Sci. USA. 82:3144-3148.

10. Segal, A. W., and O. T. G. Jones. 1978. Novel cytochrome $b$ system in phagocytic vacuoles from human granulocytes. Nature (Lond.). 276:515-517.

11. Hattori, H. 1961. Studies on the labile, stable NADI oxidase and peroxidase staining reactions in the isolated particles of horse granulocyte. Nagoya J. Med. Sci. 23:362-378.

12. Segal, A. W., R. Garcia, H. Goldstone, A. R. Cross, and O. T. Jones. 1981. Cytochrome $b-245$ of neutrophils is also present in human monocytes, macrophages and eosinophils. Biochem. J. 196:363-367.

13. Roberts, P. J., A. R. Cross, O. T. Jones, and A. W. Segal. 1982. Development of cytochrome $b$ and an active oxidase system in association with maturation of a human promyelocytic (HL-60) cell line. $J$. Cell Biol. 95:720-726.

14. Garcia, R. C., A. R. Cross, and A. W. Segal. 1986. The development of cytochrome $b-245$ in maturing human macrophages. Biochem. J. 239:647-651.

15. Segal, A. W., and O. T. Jones. 1980. Rapid incorporation of the human neutrophil plasma membrane cytochrome $b$ into phagocytic vacuoles. Biochem. Biophys. Res. Commun. 92:710-715.

16. Garcia, R. C., and A. W. Segal. 1984. Changes in the subcellular distribution of the cytochrome $b-245$ on stimulation of human neutrophils. Biochem. J. 219:233-242.

17. Segal, A. W., and O. T. Jones. 1979. The subcellular distribution and some properties of the cytochrome $b$ component of the microbicidal oxidase system of human neutrophils. Biochem. J. 182:181-188.

18. Segal, A. W., A. R. Cross, R. C. Garcia, N. Borregaard, N. H. Valerius, J. F. Soothill, and O. T. Jones. 1983. Absence of cytochrome $b$-245 in chronic granulomatous disease: a multicenter European evaluation of its incidence and relevance. N. Engl. J. Med. 308:245-251.

19. Clark, W. M. 1960. Oxidation-Reduction Potentials of Organic Systems. Williams \& Wilkins Co., Baltimore, MD.

20. Cross, A. R., O. T. Jones, A. M. Harper, and A. W. Segal. 1981. Oxidation-reduction properties of the cytochrome $b$ found in the plasma-membrane fraction of human neutrophils: a possible oxidase in the respiratory burst. Biochem. J. 194:599-606.

21. Wood, P. M. 1987. The two redox potentials for oxygen reduction to superoxide. Trends Biochem. Sci. 12:250-251.

22. Poole, R. K. 1983. Bacterial cytochrome oxidases: a structurally and functionally diverse group of electron-transfer proteins. Biochim. Biophys. Acta. 726:205-243.

23. Cross, A. R., F. K. Higson, O. T. Jones, A. M. Harper, and A. W. Segal. 1982. The enzymic reduction and kinetics of oxidation of cytochrome $b-245$ of neutrophils. Biochem. J. 204:479-485.

24. Babior, B. M. 1987. The respiratory burst oxidase. Trends Biochem. Sci. 12:241-243.

25. Cross, A. R., J. F. Parkinson, and O. T. Jones. 1985. Mechanism of the superoxide-producing oxidase of neutrophils: $\mathrm{O}_{2}$ is necessary for the fast reduction of cytochrome $b-245$ by NADPH. Biochem. J. 226:881-884. 
26. Harper, A. M., M. J. Dunne, and A. W. Segal. 1984. Purification of cytochrome $b-245$ from human neutrophils. Biochem. J. 219:519-527.

27. Pember, S. O., B. L. Heyl, J. M. Jr. Kinkade, and J. D. Lambeth. 1984. Cytochrome $b 558$ from (bovine) granulocytes: partial purification from Triton X-114 extracts and properties of the isolated cytochrome. J. Biol. Chem. 259:10590-10595.

28. Lutter, R., M. L. J. van Schaik, R. van Zwieten, R. Ever, D. Roos, and M. N. Hamers. 1985. Purification and partial characterisation of the b-type cytochrome from human polymorphonuclear leukocytes. J. Biol. Chem. 260:2237-2244.

29. Serra, M. C., P. Bellavite, A. Davoli, J. V. Bannister, and F. Rossi. 1984. Isolation from neutrophil membranes of a complex containing active NADPH oxidase and cytochrome $b-245$. Biochim. Biophys. Acta. 788:138-146.

30. Harper, A. M., M. F. Chaplin, and A. W. Segal. 1985. Cytochrome $b-245$ from human neutrophils is a glycoprotein. Biochem. J. 227:783-788.

31. Segal, A. W. 1987. Absence of both cytochrome $b-245$ subunits from neutrophils in X-linked chronic granulomatous disease. Nature (Lond.). 326:88-91.

32. Parkos, C. A., R. A. Allen, C. G. Cochrane, and A. J. Jesaitis. 1987. Purified cytochrome $b$ from human granulocyte plasma membrane is composed of two polypeptides with relative molecular weights of 91,000 and 22,000. J. Clin. Invest. 80:732-742.

33. Parkos, C. A., R. A. Allen, C. G. Cochrane, and A. J. Jesaitis. 1988. The quaternary structure of the plasma membrane b-type cytochrome of human granulocytes. Biochim. Biophys. Acta. 932:71-83.

34. Royer-Pokora, B., L. M. Kunkel, A. P. Monaco, S. C. Goff, P. E. Newburger, R. L. Baehner, F. S. Cole, J. T. Curnutte, and S. H. Orkin. 1986. Cloning the gene for an inherited human disorderchronic granulomatous disease-on the basis of its chromosomal location. Nature (Lond.). 322:32-38.

35. Parkos, C. A., M. C. Dinauer, L. E. Walker, R. A. Allen, A. J. Jesaitis, and S. H. Orkin. 1988. Primary structure and unique expression of the 22-kilodalton light chain of human neutrophil cytochrome b. Proc. Natl. Acad. Sci. USA. 85:3319-3323.

36. Newburger, P. E., R. A. B. Ezekowitz, C. Whitney, J. Wright, and S. H. Orkin. 1988. Induction of phagocyte cytochrome $b$ heavy chain gene expression by interferon- $\gamma$. Proc. Natl. Acad. Sci. USA. 85:5215-5219.

37. Bohler, M. C., R. A. Seger, R. Mouy, E. Vilmer, A. Fischer, and C. Griscelli. 1986. A study of 25 patients with chronic granulomatous disease: a new classification by correlating respiratory burst, cytochrome $b$, and flavoprotein. J. Clin. Immunol. 6:136-145.

38. Ohno, Y., E. S. Buescher, R. Roberts, J. A. Metcalf, and J. I. Gallin. 1986. Reevaluation of cytochrome $b$ and flavin adenine dinucleotide in neutrophils from patients with chronic granulomatous disease and description of a family with probable autosomal recessive inheritance of cytochrome b deficiency. Blood. 67:1132-1138.

39. Weening, R. S., L. Corbeel, M. de Boer, R. Lutter, R. van Zwieten, and D. Roos. 1985. Cytochrome $b$ deficiency in an autosomal form of chronic granulomatous disease: a third form of chronic granulomatous disease recognized by monocyte hybridization. J. Clin. Invest. 75:915-920.

40. Kunkel, L. M., A. P. Monaco, W. Middlesworth, H. D. Ochs, and S. A. Latt. 1985. Specific cloning of DNA fragments absent from the DNA of a male patient with an X chromosome deletion. Proc. Natl. Acad. Sci. USA. 82:4778-4782.

41. Teahan, C., P. Rowe, P. Parker, N. Totty, and A. W. Segal. 1987. The X-linked chronic granulomatous disease gene codes for the beta-chain of cytochrome b-245. Nature (Lond.). 327:720-721.

42. Dinauer, M. C., S. H. Orkin, R. Brown, A. J. Jesaitis, and C. A. Parkos. 1987. The glycoprotein encoded by the X-linked chronic granulomatous disease locus is a component of the neutrophil cytochrome $b$ complex. Nature (Lond.). 327:717-720.

43. Borregaard, N., A. R. Cross, T. Herlin, O. T. Jones, and A. W. Segal. 1983. A variant form of $\mathrm{X}$-linked chronic granulomatous dis- ease with normal nitroblue tetrazolium slide test and cytochrome b. Eur. J. Clin. Invest. 13:243-248.

44. Newburger, P. E., F. W. Luscinskas, T. Ryan, C. J. Beard, J. Wright, E. R. Simons, and A. I. Tauber. 1986. Variant chronic granulomatous disease: modulation of the neutrophil defect by severe infection. Blood. 68:914-919.

45. Cagan, R. H., and M. L. Karnovsky. 1964. Enzymic basis of the respiratory stimulation during phagocytosis. Nature (Lond.). 204:255-257.

46. Light, D. R., C. Walsh, A. M. O Callaghan, E. J. Goetzl, and A. I. Tauber. 1981. Characteristics of the cofactor requirements for the superoxide-generating NADPH oxidase of human polymorphonuclear leukocytes. Biochemistry. 20:1468-1476.

47. Cross, A. R., and O. T. Jones. 1986. The effect of the inhibitor diphenylene iodonium on the superoxide-generating system of neutrophils: specific labelling of a component polypeptide of the oxidase. Biochem. J. 237:111-116.

48. Cross, A. R., O. T. Jones, R. Garcia, and A. W. Segal. 1982. The association of FAD with the cytochrome $b-245$ of human neutrophils. Biochem. J. 208:758-763.

49. Cross, A. R., J. F. Parkinson, and O. T. Jones. 1984. The superoxide-generating oxidase of leucocytes. NADPH-dependent reduction of flavin and cytochrome $b$ in solubilized preparations. Biochem. J. 223:337-344.

50. Gabig, T. G., and B. A. Lefker. 1984. Catalytic properties of the resolved flavoprotein and cytochrome $b$ components of the NADPHdependent $\mathrm{O}_{2}^{-}$-generating oxidase from human neutrophils. Biochem. Biophys. Res. Commun. 118:430-436.

51. Kakinuma, K., M. Kaneda, T. Chiba, and T. Ohnishi. 1986. Electron spin resonance studies on a flavoprotein in neutrophil plasma membranes: redox potentials of the flavin and its participation in NADPH oxidase. J. Biol. Chem. 261:9426-9432.

52. Doussiere, J., and P. V. Vignais. 1985. Purification and proper-

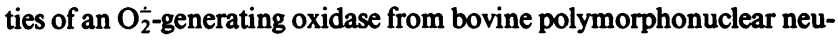
trophils. Biochemistry. 24:7231-7239.

53. Kakinuma, K., Y. Fukuhara, and M. Kaneda. 1987. The respiratory burst oxidase of neutrophils: separation of an FAD enzyme and its characterization. J. Biol. Chem. 262:12316-12322.

54. Doussiere, J., F. Laporte, and P. V. Vignais. 1986. Photolabeling of a $\mathrm{O}_{2}^{-}$-generating protein in bovine polymorphonuclear neutrophils by an arylazido NADP ${ }^{+}$analog. Biochem. Biophys. Res. Commun. 139:85-93.

55. Kojima, H., K. Takahashi, F. Sakane, and J. Koyama. 1987. Purification and characterization of NADPH-cytochrome $c$ reductase from porcine polymorphonuclear leukocytes. J. Biochem. (Tokyo). 102:1083-1088.

56. Umei, T., K. Takeshige, and S. Minakami. 1987. NADPHbinding component of the superoxide-generating oxidase in unstimulated neutrophils and the neutrophils from the patients with chronic granulomatous disease. Biochem. J. 243:467-472.

57. Gabig, T. G., and B. A. Lefker. 1984. Deficient flavoprotein component of the NADPH-dependent $\mathrm{O}_{2}^{-}$-generating oxidase in the neutrophils from three male patients with chronic granulomatous disease. J. Clin. Invest. 73:701-705.

58. Heyworth, P. G., C. F. Shrimpton, and A. W. Segal. 1989. Localisation of the $47 \mathrm{kDa}$ phosphoprotein involved in the respiratory burst NADPH oxidase of phagocytic cells: evidence for its translocation from the cytosol to plasma membrane. Biochem. J. In press.

59. Segal, A. W., and O. T. G. Jones. 1980. Absence of cytochrome b reduction in stimulated neutrophils from both female and male patients with chronic granulomatous disease. FEBS (Fed. Eur. Biochem. Soc.) Lett. 110:111-114.

60. Gennaro, R., C. Florio, and D. Romeo. 1985. Activation of protein kinase $C$ in neutrophil cytoplasts: localization of protein substrates and possible relationship with stimulus-response coupling. FEBS (Fed. Eur. Biochem. Soc.) Lett. 180:185-190.

61. Segal, A. W., P. G. Heyworth, S. Cockcroft, and M. M. Barrowman. 1985. Stimulated neutrophils from patients with autosomal 
recessive chronic granulomatous disease fail to phosphorylate a $M_{\mathrm{r}}$ 44,000 protein. Nature (Lond.). 316:547-549.

62. Heyworth, P. G., and A. W. Segal. 1986. Further evidence for the involvement of a phosphoprotein in the respiratory burst oxidase of human neutrophils. Biochem. J. 239:723-731.

63. Nunoi, H., D. Rotrosen, J. I. Gallin, and H. L. Malech. 1988. Two forms of autosomal chronic granulomatous disease lack distinct neutrophil cytosol factors. Science (Wash. DC). 242:1298-1301.

64. Kramer, I. M., A. J. Verhoeven, R. L. van der Bend, R. S. Weening, and D. Roos. 1988. Purified protein kinase C phosphorylates a 47-kDa protein in control neutrophil cytoplasts but not in neutrophil cytoplasts from patients with the autosomal form of chronic granulomatous disease. J. Biol. Chem. 263:2352-2357.

65. Shirley, P. S., D. A. Bass, C. J. Lees, J. W. Parce, and B. M. Waite. 1984. Co-localization of superoxide generation and NADP formation in plasma membrane fractions from human neutrophils. Inflammation. 8:323-335.

66. Caldwell, S. E., C. E. McCall, C. L. Hendricks, P. A. Leone, D. A. Bass, and L. C. Phail. 1988. Coregulation of NADPH oxidase activation and phosphorylation of a 48-kD protein(s) by a cytosolic factor defective in autosomal recessive chronic granulomatous disease. J. Clin. Invest. 81:1485-1496.

67. Crawford, D. R., and D. L. Schneider. 1982. Identification of ubiquinone-50 in human neutrophils and its role in microbicidal events. J. Biol. Chem. 257:6662-6668.

68. Gabig, T. G., and B. A. Lefker. 1985. Activation of the human neutrophil NADPH oxidase results in coupling of electron carrier function between ubiquinone-10 and cytochrome b559. J. Biol. Chem. 260:3991-3995.

69. Cross, A. R., O. T. Jones, R. Garcia, and A. W. Segal. 1983. The subcellular localization of ubiquinone in human neutrophils. Biochem. J. 216:765-768.

70. Babior, B. M. 1988. The respiratory burst. Ann. Intern. Med. 109:127-142.

71. Bromberg, Y., and E. Pick. 1985. Activation of NADPH-dependent superoxide production in a cell-free system by sodium dodecyl sulfate. J. Biol. Chem. 260:13539-13545.

72. McPhail, L. C., P. S. Shirley, C. C. Clayton, and R. Snyderman. 1985. Activation of the respiratory burst enzyme from human neutrophils in a cell-free system. J. Clin. Invest. 75:1735-1739.

73. Curnutte, J. T. 1985. Activation of human neutrophil nicotinamide adenine dinucleotide phosphate, reduced (triphosphopyridine nucleotide, reduced) oxidase by arachidonic acid in a cell-free system. J. Clin. Invest. 75:1740-1743.

74. Gabig, T. G., D. English, L. P. Akard, and M. J. Schell. 1987. Regulation of neutrophil NADPH oxidase activation in a cell-free system by guanine nucleotides and fluoride: evidence for participation of a pertussis and cholera toxin-insensitive $\mathrm{G}$ protein. J. Biol. Chem. 262:1685-1690.

75. Curnutte, J. T., R. Kuver, and P. J. Scott. 1987. Activation of neutrophil NADPH oxidase in a cell-free system: partial purification of components and characterization of the activation process. J. Biol. Chem. 262:5563-5569.

76. Babior, B. M., R. Kuver, and J. T. Curnutte. 1988. Kinetics of activation of the respiratory burst oxidase in a fully soluble system from human neutrophils. J. Biol. Chem. 263:1713-1718.

77. Clark, R. A., K. G. Leidal, D. W. Pearson, and W. M. Nauseef. 1987. NADPH oxidase of human neutrophils: subcellular localization and characterization of an arachidonate-activatable superoxide-generating system. J. Biol. Chem. 262:4065-4074.

78. Curnutte, J. T. 1988. Classification of chronic granulomatous disease. In Haematology/Oncology Clinics of North America. Phagocytic defects Volume 11: Abnormalities of the Respiratory Burst. J. T. Curnutte, editor. W. B. Saunders Co., Philadelphia. 241-252.
79. Sechler, J. M. G., H. L. Malech, C. J. White, and J. I. Gallin. 1988. Recombinant human interferon-g reconstitutes defective phagocyte function in patients with chronic granulomatous disease of childhood. Proc. Natl. Acad. Sci. USA. 85:4874-4878.

80. Volpp, D. D., W. M. Nauseef, and R. A. Clark. 1988. Two cytosolic neutrophil oxidase components absent in autosomae chronic granulomatous disease. Science (Wash. DC). 242:1295-1297.

81. Sakane, F., H. Kojima, K. Takahashi, and J. Koyama. 1987. Porcine polymorphonuclear leukocyte NADPH-cytochrome $c$ reductase generates superoxide in the presence of cytochrome $b 559$ and phospholipid. Biochem. Biophys. Res. Commun. 147:71-77.

82. Berton, G., L. Zani, M. A. Cassatella, and F. Rossi. 1986. Gamma interferon is able to enhance the oxidative metabolism of human neutrophils. Biochem. Biophys. Res. Commun. 138:12761282.

83. Ezekowitz, R. A. B., M. C. Dinauer, H. S. Jaffe, S. H. Orkin, and P. E. Newburger. 1988. Partial correction of the phagocyte defect in patients with X-linked chronic granulomatous disease by subcutaneous interferon gamma. N. Engl. J. Med. 319:146-151.

84. Cassatella, M. A., L. Hartman, B. Perussia, and G. Trinchieri. 1989. Tumor necrosis factor and immune interferon synergistically induce cytochrome b-245 heavy chain gene expression and NADPH oxidase in human leukemic myeloid cells. J. Clin. Invest. In press.

85. McPhail, L. C., and R. Snyderman. 1983. Activation of the respiratory burst enzyme in human polymorphonuclear leukocytes by chemoattractants and other soluble stimuli: evidence that the same oxidase is activated by different transductional mechanisms. J. Clin. Invest. 72:192-200.

86. Sha'afi, R. I., T. F. P. Molski, J. Gomez-Cambronero, and C.-K. Huang. 1988. Dissociation of the 47-kilodalton protein phosphorylation from degranulation and superoxide production in neutrophils. J. Leukocyte. Biol. 43:18-27.

87. Jacques, Y. V., and D. F. Bainton. 1978. Changes in $\mathrm{pH}$ within the phagocytic vacuoles of human neutrophils and monocytes. Lab. Invest. 39:179-185.

88. Mandell, G. L. 1970. Intraphagosomal pH of human polymorphonuclear neutrophils. Proc. Soc. Exp. Biol. Med. 134:447-449.

89. Segal, A. W., M. Geisow, R. Garcia, A. Harper, and R. Miller. 1981. The respiratory burst of phagocytic cells is associated with a rise in vacuolar pH. Nature (Lond.). 290:406-409.

90. Cech, P., and R. I. Lehrer. 1984. Phagolysosomal pH of human neutrophils. Blood. 63:88-95.

91. Henderson, L. M., J. B. Chappell, and O. T. Jones. 1987. The superoxide-generating NADPH oxidase of human neutrophils is electrogenic and associated with an $\mathrm{H}+$ channel. Biochem. J. 246:325329.

92. Roos, D., A. A. Voetman, and L. J. Meerhof. 1983. Functional activity of enucleated human polymorphonuclear leukocytes. J. Cell Biol. 97:368-377.

93. Odell, E. W., and A. W. Segal. 1988. The bactericidal effects of the respiratory burst and the myeloperoxidase system isolated in neutrophil cytoplasts. Biochim. Biophys. Acta. 971:266-274.

94. Klebanoff, S. J., and R. A. Clark. 1978. The Neutrophil: Function and Clinical Disorders. Elsevier North-Holland, New York.

95. Odeberg, H., and I. Olsson. 1975. Antibacterial activity of cationic proteins from human granulocytes. J. Clin. Invest. 56:11181124.

96. Quie, P. G., J. G. White, B. Holmes, and R. A. Good. 1967. In vitro bactericidal capacity of human polymorphonuclear leukocytes: diminished activity in chronic granulomatous disease of childhood. $J$. Clin. Invest. 46:668-679.

97. Landing, B. H., and H. S. Shirkey. 1957. A syndrome of recurrent infection and infiltration of viscera by pigmented lipid histiocytes. Pediatrics. 20:431-438. 